\title{
Can 3D Electron Diffraction Provide Accurate Atomic Structures of Metal- Organic Frameworks?
}

\author{
Zhehao Huang, ${ }^{\text {a a }}$ Meng Ge, ${ }^{\text {a }}$ Francesco Carraro, ${ }^{\mathrm{b}}$ Christian Doonan, ${ }^{\mathrm{c}}$ Paolo Falcaro, ${ }^{\mathrm{b}}$ Xiaodong Zou, ${ }^{* a}$ \\ aDepartment of Materials and Environmental Chemistry, Stockholm University, 10691 Stockholm, Sweden \\ bInstitute of Physical and Theoretical Chemistry, Graz University of Technology, Stremayrgasse 9, 8010 Graz, Austria \\ ${ }^{\mathrm{c}}$ Department of Chemistry and the Centre for Advanced Nanomaterials, The University of Adelaide, Adelaide, 5005 South \\ Australia, Australia \\ *Corresponding author: zhehao.huang@mmk.su.se; $\underline{x z o u @ m m k . s u . s e}$
}

\begin{abstract}
:
Many framework materials such as metal-organic frameworks (MOF) or porous coordination polymers (PCPs) are synthesized as polycrystalline powders, which are too small for structure determination by single crystal X-ray diffraction (SCXRD). Here, we show that a three-dimensional (3D) electron diffraction method, namely continuous rotation electron diffraction (cRED), can be used for ab initio structure determination of such materials. As an example, we present a complete structural analysis of a biocomposite, denoted BSA@ZIF-C, where Bovin Serum Albumin (BSA) was encapsulated in a zeolitic imidazolate framework (ZIF). Low electron dose was combined with ultrafast cRED data collection to minimize electron beam damage of the sample. We demonstrate that the atomic structure obtained by cRED is as reliable and accurate as that obtained by single crystal X-ray diffraction. The high accuracy and fast data collection open new opportunities for investigation of cooperative phenomena in framework structures at atomic level.
\end{abstract}

\section{INTRODUCTION}

Metal-organic frameworks (MOFs) or porous coordination polymers (PCPs) offer large surface areas, tuneable pore structures, adjustable chemical functionality and structural flexibility. ${ }^{1,2}$ Because of these unique structural properties, they have tremendous potential in a wide range of applications such as catalysis, gas storage and separation, ion exchange, bio-medical and bio-technological applications. ${ }^{3-9}$ Notably, under different synthesis conditions, various MOF structures with different topologies and pore sizes can be obtained using the same metal ions and organic linkers. In many cases, MOFs undergo several stages of structural transformations during the synthesis. For example, a combination of 2methylimidazole (HmIM) and Zn(II) cations could produce zeolitic imidazolate framework (ZIFs) with different topologies, such as sodalite (sod), diamondoid (dia), katsenite ( $k a t)$, and a layered ZIF-L ${ }^{10-13}$. In addition, many MOFs are flexible and undergo structural changes when interact with other species, known by the breathing and swelling phenomena associated with the host-guest interactions. ${ }^{14-18}$ The structural changes can also be triggered by other external stimuli, such as photochemical, thermal, and mechanical 
changes. ${ }^{19-22}$ One the other hand, many MOF frameworks are rigid, and incorporation of guest species or defects in MOF structures does not necessarily involve crystallographic structural changes. ${ }^{23-28}$ In all cases, it is essential to know the exact arrangements of atoms in the material in order to understand the properties and functionalities of the material as well as the host-guest interactions. Therefore ab initio structure determination of the material is crucial.

Single crystal X-ray diffraction (SCXRD) has been the most powerful method for structure determination of crystalline materials, including MOFs ${ }^{29-31}$. However, structure determination by SCXRD requires large $(>5 \mu \mathrm{m})$ and well-ordered crystals, which is sometimes difficult to synthesize. Furthermore, experiments for studying cooperative phenomena may also destroy the crystallinity of the MOFs. As a consequence, many nano- and micron-sized MOFs have generally been discarded because of the difficulties in their structure determination. Thus, methods that can facilitate structure determination from polycrystalline powders would be of great importance for the development new MOF materials and studies of the cooperative phenomena. Although powder X-ray diffraction (PXRD) can be an alternative method for studying polycrystalline MOFs, ab initio structure determination by PXRD is still very challenging, especially when diffraction peaks overlap and samples contain multiple phases. The peak overlapping becomes more severe for structures with large unit cell parameters and crystals with small sizes and containing defects.

Single crystal electron diffraction can be obtained from crystals that are too small to be studied by SCXRD. Although ab initio structure analysis by using electron diffraction was demonstrated earlier ${ }^{32-}$ ${ }^{34}$, the major revolution was made during the past decade by the development of new $3 \mathrm{D}$ electron diffraction methods ${ }^{35,36}$. Several groups independently developed 3D single-crystal electron diffraction methods and software for semi-automatic data collection on standard TEMs. These include rotation electron diffraction (RED) by combining step-wise fine beam tilt and coarse crystal rotation ${ }^{36,37}$, electron diffraction tomography by step-wise crystal rotation without or with electron precession (automated diffraction tomography $(\mathrm{ADT})^{35,38}$, or precession-assisted electron diffraction tomography $(\mathrm{PEDT})^{39}$ ), and microcrystal electron diffraction (MicroED) by step-wise crystal rotation ${ }^{40}$. Continuous rotation electron diffraction (cRED) was later developed independently by several groups ${ }^{41-44}$, also known as IEDT ${ }^{42}$ and MicroED ${ }^{43}$.

3D electron diffraction (ED) methods have shown to be powerful for ab initio structure determination of MOFs from nano- and micron-sized crystals ${ }^{45-51}$. Because of the strong interactions of electrons with matter, 3D single crystal electron diffraction can be obtained from nanocrystals that are $10^{8}$ times smaller in volumes than those needed for single crystal X-ray diffraction. On the other hand, the strong interaction also leads to multiple scattering of electrons in the crystal, which makes the intensities of reflections dynamical and deviating from the kinematical intensities. Although it is possible to solve and refine the structures using 3D ED data, the discrepancy between the kinematical intensities calculated from the structure model and the dynamical intensities obtained experimentally results in relatively high R-values after the structure refinement $(>0.15)$. It is thus important to compare the structural models obtained by 3D ED with those obtained by SCXRD to know what accuracy a structure determination by 3D ED can 
achieve. Although this has been studied on stable crystals such as zeolites and metal oxides ${ }^{52,53}$, it has not yet been applied to MOFs.

Here, we report an ab initio structure determination of a ZIF biocomposite by continuous rotation electron diffraction (cRED). The ZIF biocomposite was synthesized from $\mathrm{Zn}(\mathrm{OAc})_{2} \cdot 2 \mathrm{H}_{2} \mathrm{O}, \mathrm{HmIM}$, Bovine Serum Albumin (BSA) in water ${ }^{54}$, denoted BSA@ZIF-C. It was considered as a new ZIF phase. The structure was solved and refined using cRED data, which turns to be the same as that of ZIF-CO -1 previously determined by SCXRD using synchrotron data ${ }^{55}$. The results of structure determination by cRED were compared to those by SCXRD.

\section{EXPERIMENTAL SECTION}

Synthesis. The BSA@ZIF-C was discovered during the study of phase dependent encapsulation and release of ZIF-based biocomposites. ${ }^{54}$ In detail, $300 \mu \mathrm{L}$ of a $440 \mathrm{mM}$ aqueous solution of 2methylimidazole, HmIM (TCI Chemicals), $60 \mu \mathrm{L}$ of a $36 \mathrm{mg} / \mathrm{ml}$ aqueous solution of BSA (lyophilized powder, Sigma-Aldrich) and $1140 \mu \mathrm{L}$ of deionized water were mixed in a $2 \mathrm{ml}$ plastic centrifuge tube for 1 minute. Then, this solution was added to $500 \mu \mathrm{L}$ of a $80 \mathrm{mM}$ aqueous solution of $\mathrm{Zn}(\mathrm{OAc})_{2} \cdot 2\left(\mathrm{H}_{2} \mathrm{O}\right)$ (EMSURE, Merck). The reaction mixture was left under static conditions (no shaking and no stirring) at RT for $24 \mathrm{~h}$. After this reaction time, the biocomposite was isolated via centrifugation (13000 rpm for 5 min; centrifuge used: Eppendorf 5425) and the supernatant was discarded. The obtained powder pellet was then re-suspended in deionized water $(1.5 \mathrm{~mL})$ using a vortex mixer $(3000 \mathrm{rpm}, 1 \mathrm{minute}$, VELP Scientifica ZX3) and the centrifugation step (13000 rpm, $5 \mathrm{~min}$ ) was repeated. This washing procedure was repeated 6 times using deionized (DI) water. Finally, the recovered powders were air-dried for $48 \mathrm{~h}$ at RT. The PXRD pattern is shown in Figure 1, which does not match any of the known ZIFs built of Zn (II) and 2-methylimidazate. The material was therefore considered to be a new ZIF, and ab initio structure determination by cRED was performed.

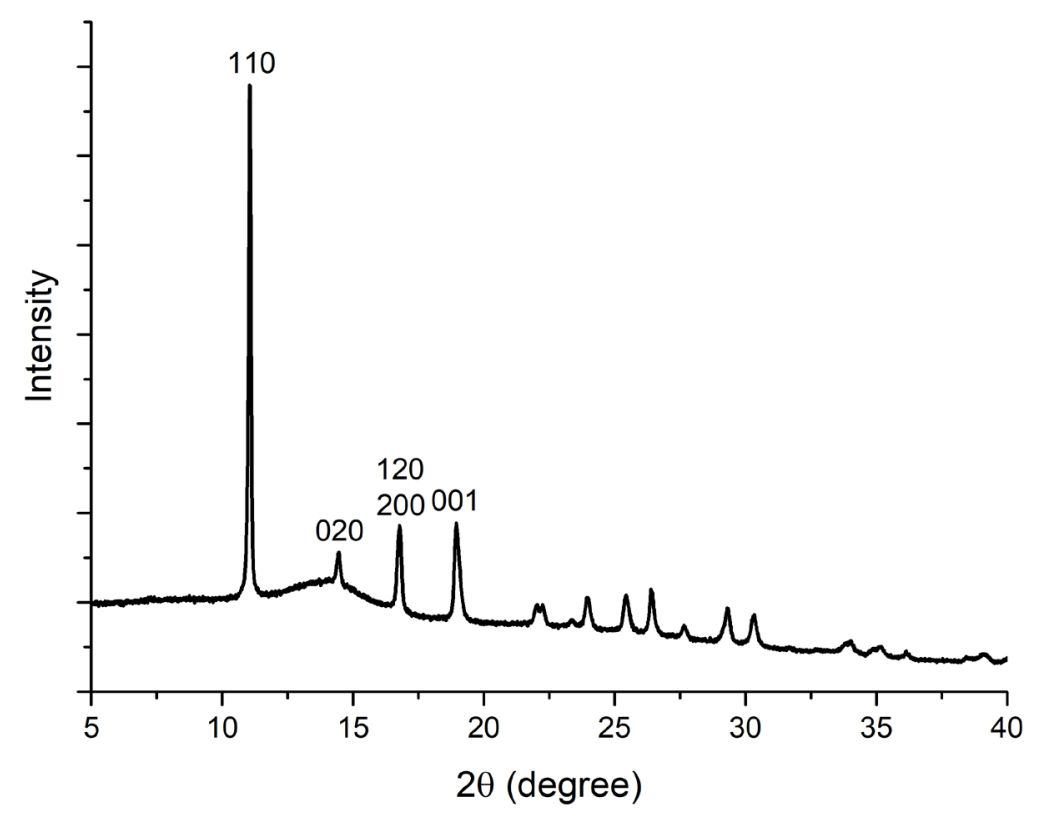

Figure 1. The PXRD pattern $(\lambda=1.5418 \AA)$ of BSA@ZIF-C. 
TEM and cRED data collection. Samples for TEM investigations were dispersed in water. A droplet of the suspension was transferred onto a carbon-coated copper grid. TEM images and cRED data were collected on a JEOL JEM2100 microscope operated at $200 \mathrm{kV}$ (Cs $1.0 \mathrm{~mm}$, point resolution $0.23 \mathrm{~nm}$ ). TEM images were recorded with a Gatan Orius 833 CCD camera (resolution 2048 x 2048 pixels, pixel size $7.4 \mu \mathrm{m}$ ). cRED data collection was controlled by using the data acquisition software Instamatic, ${ }^{41,56}$ and the electron diffraction (ED) frames were recorded with a Timepix hybrid detector QTPX-262k (512 x 512 pixels, pixel size $55 \mu \mathrm{m}$, max 120 frames/second, Amsterdam Sci. Ins.) A single-tilt tomography holder was used for the data collection, which could tilt from $-70^{\circ}$ to $+70^{\circ}$ in the TEM. The area used for cRED data collection was about $1.0 \mu \mathrm{m}$ in diameter, as defined by a selected area aperture. To minimize electron beam damage of the crystal and maximize the data quality, a low electron dose and high rotation speed were applied. The goniometer rotation speed was $0.45^{\circ}$ per second and the exposure time was 0.5 $\mathrm{s}$ per frame. A typical cRED dataset covered a crystal rotation angle of $103.3^{\circ}$ and took $3.8 \mathrm{~min}$ to collect. cRED datasets were collected from several crystals.

\section{RESULTS AND DISCUSSION}

Continuous rotation electron diffraction data collection. Different from the step-wise data collection in ADT/PEDT and RED where no crystal rotation is applied during the exposure, the crystal is rotated continuously with a constant speed during the cRED data collection (Figure 2). ${ }^{53}$ The time needed to collect a dataset is determined by the total rotation range and the rotation speed of the crystal. By using the Timepix hybrid detector, a complete cRED dataset could be collected in less than one minute, with an electron dose rate of $<0.1 \mathrm{e} \AA^{-2} \mathrm{~s}^{-1}$. This development is crucial for studies of electron beam-sensitive materials, e.g. MOFs, COFs, ZIFs, etc. In order to track the crystal movement and keep it in the beam during the continuous rotation, we developed manual ${ }^{41}$ and automated ${ }^{57}$ crystal tracking by defocusing ED frames at given intervals during crystal rotation, which are implemented in the software Instamatic ${ }^{56}$. In this way, re-centering of crystals can be achieved without stopping the crystal rotation. By taking advantage of the cRED method, the quality of datasets and the accuracy of structure determination are improved, and thereby more detailed structural information can be revealed.

3D electron diffraction data are collected at arbitrary orientations of a crystal, most of the ED frames are off the zone axes which largely minimizes the chance for multiple scattering in the crystal ${ }^{34,58}$. The RED data processing program can process $3 \mathrm{D}$ electron diffraction data collected by both step-wise and continuous rotation methods, and reconstruct the 3D reciprocal lattice (Figure 3) from which the unit cell parameters and space group can be determined ${ }^{37}$. Because cRED data collection resembles SCXRD data collection, existing software for data processing developed for SCXRD can be directly used. Many X-ray crystallographic software, such as $\mathrm{XDS}^{59,60}$, DIALS ${ }^{61,62}$, and MOSFLM ${ }^{63}$ have been adapted to integrate and extract intensity information from cRED data. The intensities are subsequently treated as kinematical input for ab initio structure solution via direct methods, charge flipping, etc. 

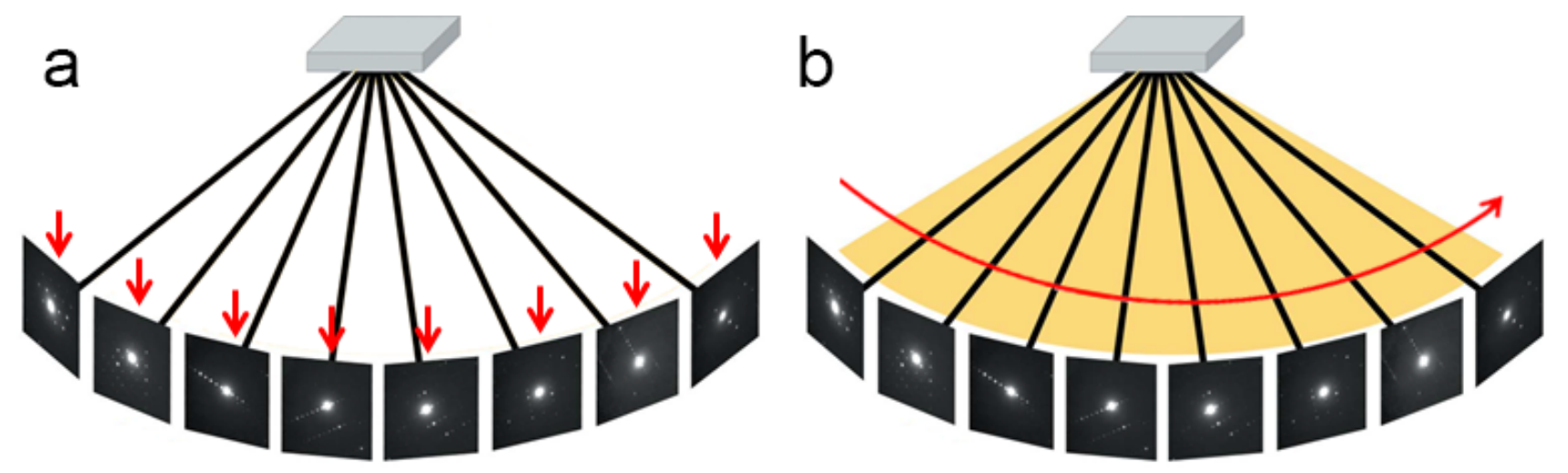

Figure 2. Illustration of stepwise and continuous rotation methods. (a) The stepwise rotation methods collect each ED frame at a certain angle. The advantage is that it is possible to track the crystal and perform re-centering and height adjustment. (b) The continuous rotation methods collect ED frames continuously. The advantages are integrated diffraction intensities can be obtained and data collection is much faster than that for step-wise rotation.

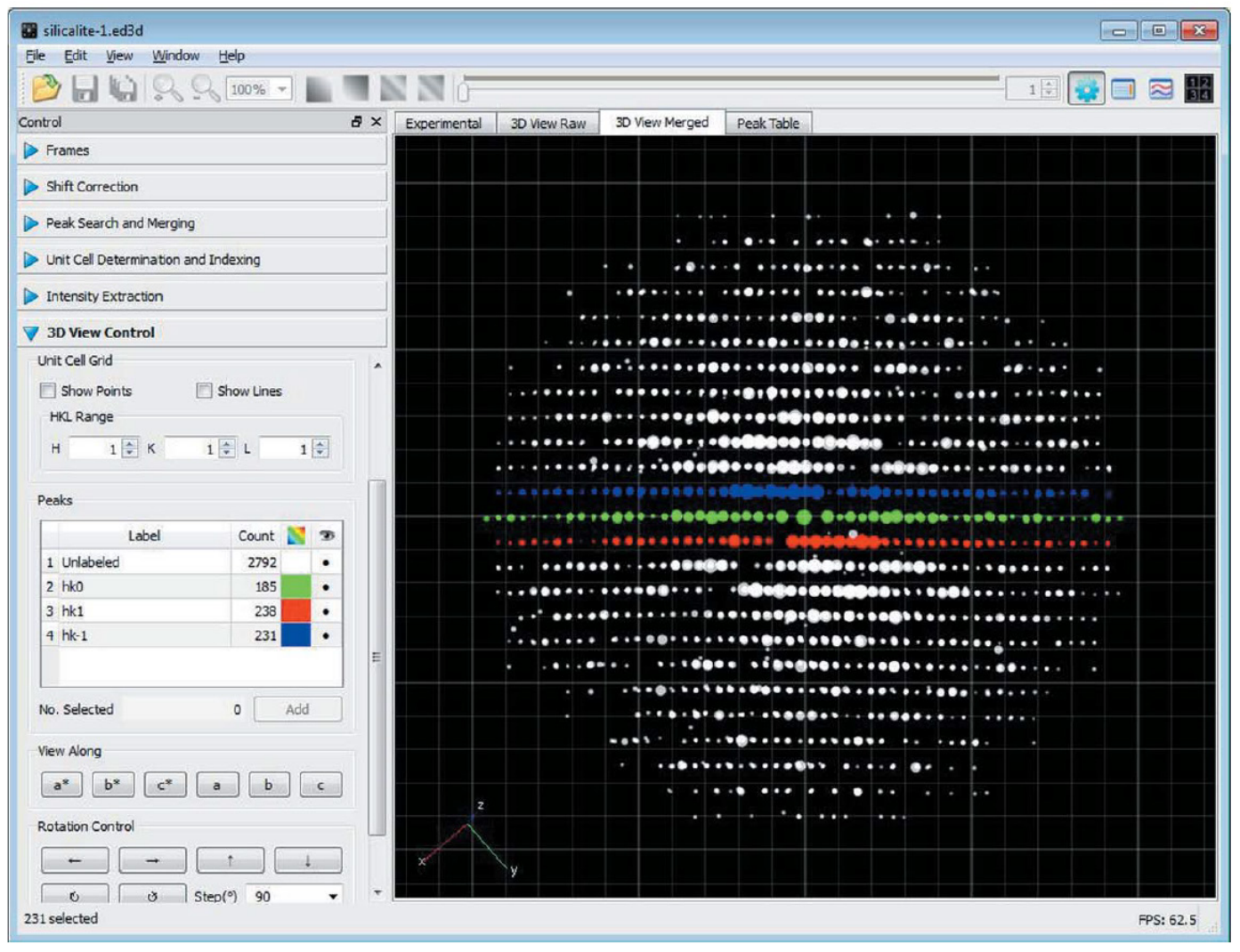

Figure 3. The graphical user interface of the RED data processing program. It shows the reconstructed 3D reciprocal lattice for a zeolite, obtained from 1472 individual ED frames. The $h k 0, h k 1$ and hk-1 layers are marked in green, red and blue, respectively ${ }^{37}$. Copyright, International Union of Crystallography.

cRED data processing and structure determination of BSA@ZIF-C. We applied $c$ RED to determine the structure of BSA@ZIF-C. The first step was to determinate the unit cell parameters and space group. $3 \mathrm{D}$ reciprocal lattice was reconstructed from the cRED data by the RED data processing program ${ }^{37}$ and is shown in Figure 4. All diffraction spots can be indexed by a single primitive lattice, confirming the single crystal nature of the BSA@ZIF-C biocomposite. The unit cell parameters were determined to be $a$ 
$=10.31 \AA, b=12.51 \AA, c=4.65 \AA, \alpha=88.8^{\circ}, \beta=89.5^{\circ}, \gamma=89.6^{\circ}$. The intensity distribution of reflections in the $3 \mathrm{D}$ reciprocal lattice indicates that the crystal is orthorhombic with the Laue class $\mathrm{mmm}$ (Figure 4). The unit cell angles $\alpha, \beta$, and $\gamma$ are near $90^{\circ}$, which also confirms the orthorhombic crystal system. The reflection conditions can be deduced from the two-dimensional (2D) slices cut at $h k 0,0 k l$, and $h 0 l$ planes; $0 k l: k=2 n ; h 0 l: h=2 n ; h 00, h=2 n ; 0 k 0: k=2 n$, which correspond to two possible space groups: Pba2 (No. 32), and Pbam (No. 55). A broad sphere is observed in addition to the sharp diffraction spots, presumably due to contribution of the BSA molecules. This indicates that BSA molecules are encapsulated in the ZIF crystal.
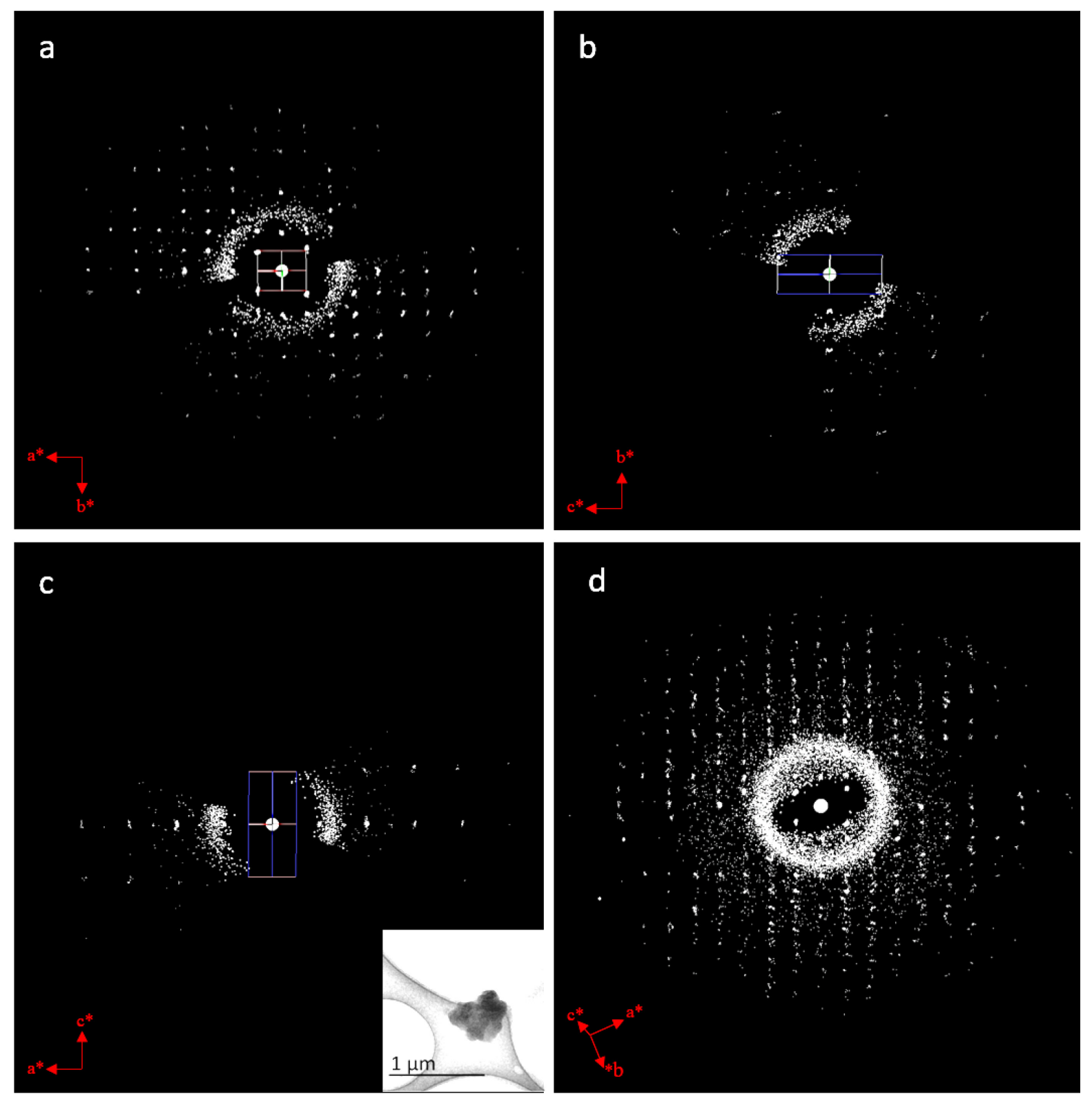

Figure 4. (a-c) 2D slices cut from the reconstructed 3D reciprocal lattice of BSA@ZIF-C showing the (a) $h k 0$, (b) $0 k l$, and (c) $h 0 l$ planes. The reflection conditions can be deduced to be $0 k l: k=2 n ; h 0 l: h=2 n ; h 00, h=2 n ; 0 k 0: k$ $=2 n$. (d) 3D reciprocal lattice of BSA@ZIF-C. All diffraction spots can be indexed by a single lattice, indicating the single crystal nature. A broad ring is observed in all ED patterns, which is probably coming from the encapsulated BSA molecules. 
The cRED dataset of BSA@ZIF-C was further processed by using the XDS package ${ }^{59,60}$, from which the unit cell parameters were refined and intensities of reflections were integrated. The cRED data reached a resolution of $1.00 \AA$ and a completeness is $79.7 \%$, which is sufficiently enough for $a b$ initio structure solution by direct methods. The framework structure of BSA@ZIF-C was solved by direct methods using the program SHELXT-2014 $4^{64,65}$ and atomic scattering factors of electrons. Both space groups Pba2 and Pbam were tested. While many atoms were too close to each other in the structure solution using Pbam, the structure determined using Pba2 was reasonable. The positions of all non- $\mathrm{H}$ atoms, including $\mathrm{Zn}, \mathrm{C}$, $\mathrm{N}$ and $\mathrm{O}$, could be found directly. The assignment of the atom types was done according to the chemical knowledge.

A good structure refinement requires high data completeness $(>90 \%)$. In order to increase the data completeness, two cRED datasets from crystals with different initial orientations were merged. The merged data reached a completeness of $94.5 \%$. During the merging step, it is important to ensure that the intensities of common reflections between the datasets have a high similarity. This is assessed via correlation coefficients $C C_{1 / 2}$ of the common reflections between the datasets ${ }^{66}$. Due to the increased number of reflections after data merging, the $R_{\text {int }}$ value increased from 0.174 to 0.280 . The final refinement was done by using SHELXL-2014, which converged to $R_{1}=0.156$. The details of data collection and structure refinement were summarized in Table 1.

Table 1. Comparison of crystallographic data and refinement details of BSA@ZIF-C by cRED and ZIF-CO ${ }_{3}-1$ by $\mathrm{SCXRD}^{55}$.

\begin{tabular}{lll}
\hline & cRED data & Synchrotron SCXRD data $^{55}$ \\
\hline No. dataset merged & 2 & 1 \\
Wavelength $(\AA)$ & $0.02508 \AA$ & $0.68890 \AA$ \\
Crystal size $\left(\mu \mathrm{m}^{3}\right)$ & $0.5 \times 0.5 \times 0.3$ & $40 \times 13 \times 7$ \\
Resolution $(\AA)$ & 1.00 & 0.79 \\
Crystal system & Orthorhombic & Orthorhombic \\
Space group & $P b a 2($ No. 21$)$ & Pba2 (No. 21) \\
Unit cell $a, b, c(\AA)$ & $10.510(2), 12.234(2), 4.6660(9)$ & $10.536(9), 12.314(10), 4.659(4)$ \\
Volume $\left(\AA^{3}\right)$ & $600.0(2)$ & $604.5(8)$ \\
Completeness $(\%)$ & 94.5 & 98.3 \\
No. reflections in $c$ RED & 1638 & 4239 \\
No. unique reflections & 575 & 1169 \\
No. observed reflections $(\mathrm{I}>$ & 320 & 1040 \\
2 sigma(I)) & & \\
$R_{1}(\mathrm{I}>2$ sigma(I)) & 0.156 & 0.043 \\
$R_{1}($ all reflections) & 0.225 & 0.049 \\
Goof & 1.272 & 1.022 \\
\hline
\end{tabular}

The structure of ZIF-C is shown in Figure 5. Surprisingly, in addition to $\mathrm{Zn}(\mathrm{II})$ cation and mIM ions, carbonate ions $\mathrm{CO}_{3}{ }^{2-}$ are also found in the structure. The structure is the same as that of $\mathrm{ZIF}-\mathrm{CO}_{3}-1$ 
previously determined by $\mathrm{SCXRD}{ }^{55}$. ZIF- $-\mathrm{CO}_{3}-1$ was previously synthesized from $\mathrm{Zn}\left(\mathrm{NO}_{3}\right)_{2} \cdot 6 \mathrm{H}_{2} \mathrm{O}$ and $\mathrm{HmIM}$ in the presence of $\mathrm{CO}_{2}$, which was either introduced in the synthesis or in situ generated via hydrolysis of N,N-dimethylformamide (DMF). The $\mathrm{CO}_{2}$ was incorporated in the framework as a carbonate $\mathrm{CO}_{3}{ }^{2-}$. The captured $\mathrm{CO}_{2}$ can be selectively released by acidifying the ZIF material. The ZIF$\mathrm{CO}_{3}-1$ framework can be regenerated in water followed by exposure to $\mathrm{CO}_{2}{ }^{55}$. In our synthesis, neither $\mathrm{CO}_{2}$ gas nor DMF was used. The incorporated carbonate was presumably derived from the $\mathrm{CO}_{2}$ dissolved in the deionized water.

The asymmetric unit consists of one $\mathrm{Zn}$ (II) cation, one mIM, and half of a carbonate ion. Each $\mathrm{Zn}$ (II) cation binds to two mIM ions to form a chain along the $b$-axis (Figures $5 \mathrm{~b}$ ). The chains are further connected by the carbonates to form a 3D framework (Figures 5). The three oxygen from each carbonate bind to four $\mathrm{Zn}(\mathrm{II})$ cations from four different chains (Figures 5a). They play a crucial role for the formation of the 3D ZIF framework. As expected, the encapsulation of BSA molecules are not ordered and cannot be located by cRED. However, it is interesting to compare the structural model with that of ZIF-CO -1 determined by SCXRD, and see whether the encapsulation of proteins affects the atomic structures.
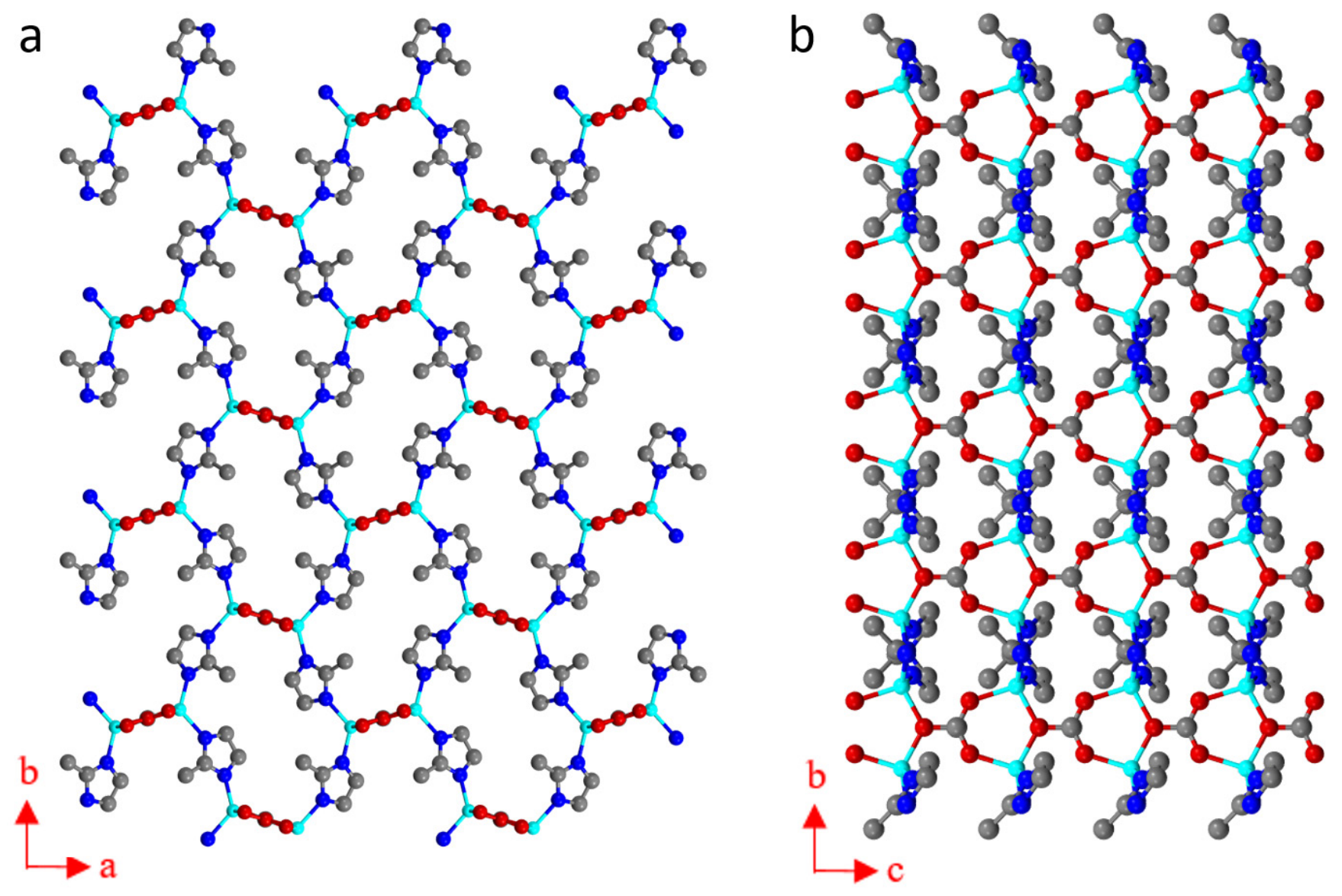

Figure 5. The structural model of BSA@ZIF-C viewed along (a) $c$-axis, and (b) $a$-axis. Blue spheres: N; red spheres: O; gray spheres: C; cyan spheres: Zn atoms. 
Comparison of refinement results and structural models obtained by $\boldsymbol{c R E D}$ and SCXRD. Electron diffraction suffers from multiple scattering which makes the intensities of reflections dynamical. However, in structure determination by cRED using software developed for SCXRD, the intensities are treated as kinematical. As a result, structure refinements against cRED data often lead to relatively high $R_{1}$ values compared with those refined against SCXRD data. In addition, electron optics of the TEM, errors in goniometer rotation as well as the sample height may introduce geometrical distortions on the cRED data, which affects the unit cell determination. It is therefore important to compare the unit cell parameters, results of crystallographic structure refinement and finally the structural models obtained by cRED and SCXRD ${ }^{55}$. Those comparisons are given in Figure 6 and Tables 1-3.

One of the obvious advantages of cRED is that only very small crystals are required. As shown in Table 1, the crystals of BSA@ZIF-C examined by cRED were $0.5 \times 0.5 \times 0.3 \mu \mathrm{m}^{3}$ in size, which is ca. 50000 times smaller than the crystal of ZIF-CO $-1\left(40 \times 13 \times 7 \mu \mathrm{m}^{3}\right.$ in size $)$ studied by SCXRD using a synchrotron source ${ }^{55}$. The resolution of the cRED data is $1.00 \AA$, lower than that of the SCXRD data $(0.79$ $\AA$ ). The lower resolution of the cRED data was not caused by beam damage, but the lower crystallinity of BSA@ZIF-C, as also shown in its PXRD pattern (Figure 1). The lower crystallinity is probably caused by the encapsulation of the BSA molecules.

The combined cRED dataset has a data completeness of $94.5 \%$ with 575 unique reflections, while the SCXRD dataset has a completeness of $98.3 \%$ with 1169 unique reflections. The unit cell parameters obtained by cRED are very close to those determined by SCXRD, and only differ by $0.03(1)(0.3 \%)$, $0.08(1)(0.6 \%)$ and $0.007(5)(0.2 \%) \AA$ for $a, b$ and $c$, respectively. This shows that the unit cell parameters obtained by cRED can be as accurate as those obtained by SCXRD, and the encapsulation of BSA did not change the unit cell parameters.

Even though the $R_{l}$ value for the structure refinement of BSA@ZIF-C against the cRED data (0.227) is much higher compared to that of ZIF- $\mathrm{CO}_{3}-1$ against SCXRD data (0.049), the two structural models are very similar, as shown in Figure 6 and Table 2. The position of the heavy $\mathrm{Zn}$ atom deviates by $0.06(1) \AA$, while positions of the light $\mathrm{N}, \mathrm{C}$, and $\mathrm{O}$ atoms deviate by 0.07(3) $\AA$ on average. The $\mathrm{C} 7, \mathrm{O} 1$ and $\mathrm{O} 2$ positions belonging to the carbonate differ on average by 0.03(2) $\AA$ for the two models. The largest deviation of $0.13(5) \AA$ occurs on the methyl group (C6). The deviations are comparable to those more stable materials such as zeolite ZSM-5 (0.07(3) $\AA$ with $0.87 \AA$ resolution) and $\mathrm{FeSeO}_{3} \mathrm{~F}$ (0.03(1) $\AA$ with $0.78 \AA$ resolution). ${ }^{53}$ The bond lengths and angles of the two models also show good agreements (Table 3). The average deviation is 0.04(3) $\AA$ for the bond lengths and $4(3)^{\circ}$ for the bond angles. The anisotropic atomic displacement parameters are also reasonable. This shows that despite the lower crystallinity of the BSA@ZIF-C and high $R_{l}$-values, the structural model obtained from cRED data is consistent with that of ZIF-CO -1 obtained by SCXRD. This suggests that the high $R_{1}$-values are mainly caused by dynamical effects. The encapsulation of BSA did not alter the framework structure of $\mathrm{ZIF}-\mathrm{CO}_{3}-1$. 

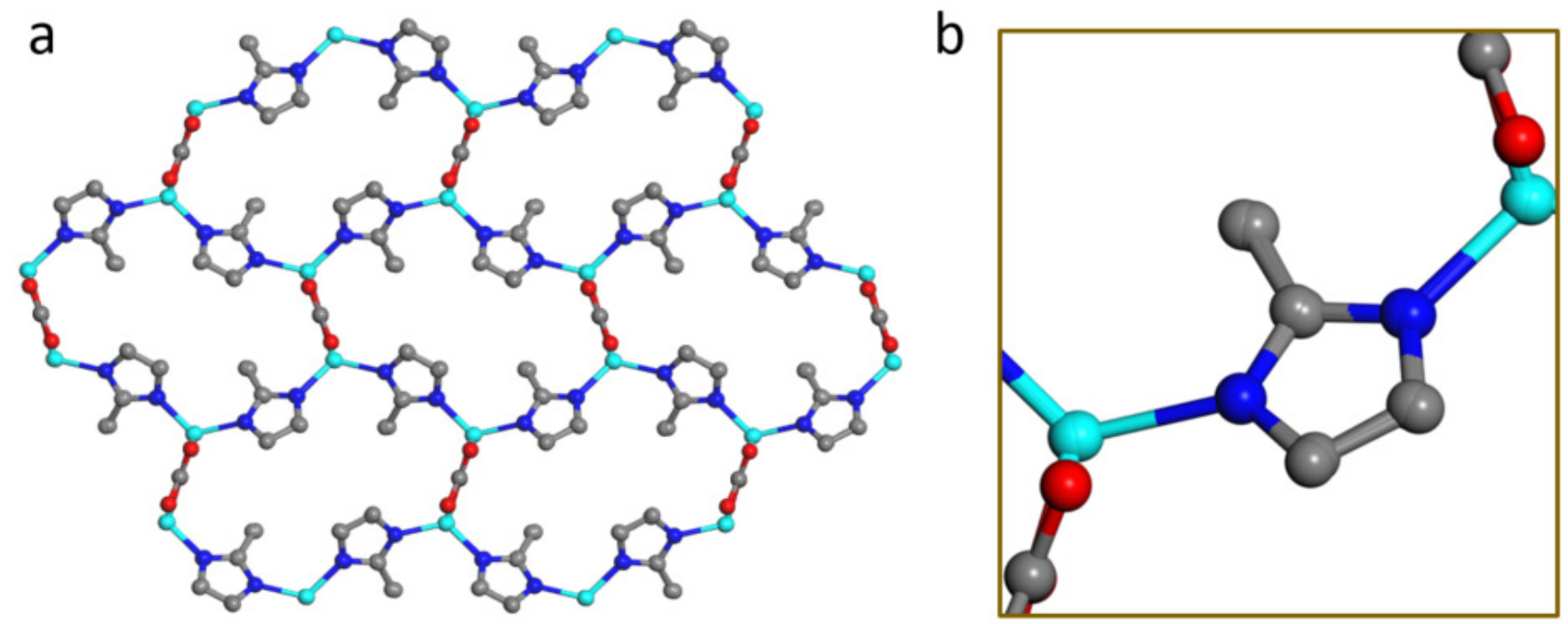

Figure 6. Comparison between the structural model of BSA@ZIF-C refined from cRED data and that of ZIF-CO ${ }_{3}^{-}$ 1 from SCXRD data. (a) The structures are stacked together, viewed along $c$-axis. (b) An enlarged figure showing the mIM linker and $\mathrm{Zn}(\mathrm{II})$ cations, illustrating the accuracy of the model obtained from the cRED data. Blue spheres: N; red spheres: O; gray spheres: C; cyan spheres: Zn atoms.

Table 2. Comparison of the atomic positions of BSA@ZIF-C refined against cRED data with those of ZIF-CO $\mathrm{CO}_{3}-1$ refined against synchrotron SCXRD data.

\begin{tabular}{|c|c|c|c|c|c|c|c|}
\hline \multirow{2}{*}{$\begin{array}{l}\text { Atom } \\
\mathrm{Zn} 1\end{array}$} & \multicolumn{3}{|c|}{$\mathrm{x}, \mathrm{y}, \mathrm{z}$ coordinates from $\mathrm{cRED}$} & \multicolumn{3}{|c|}{$\mathrm{x}, \mathrm{y}, \mathrm{z}$ coordinates from SCXRD } & \multirow{2}{*}{$\begin{array}{l}\text { Difference }(\AA) \\
0.06(1)\end{array}$} \\
\hline & $0.0453(10)$ & $0.1357(9)$ & $0.947(4)$ & $0.05069(5)$ & $0.13664(4)$ & $0.94752(16)$ & \\
\hline $\mathrm{N} 2$ & $-0.089(3)$ & $0.246(2)$ & $1.000(7)$ & $-0.0818(5)$ & $0.2476(4)$ & $1.0047(10)$ & $0.08(3)$ \\
\hline N3 & $0.229(3)$ & $0.172(2)$ & $1.039(7)$ & $0.2299(5)$ & $0.1726(4)$ & $1.0227(9)$ & $0.08(3)$ \\
\hline $\mathrm{C} 1$ & $0.289(3)$ & $0.259(2)$ & $0.918(6)$ & $0.2964(4)$ & $0.2548(4)$ & $0.9157(15)$ & $0.09(3)$ \\
\hline $\mathrm{C} 4$ & $0.309(3)$ & $0.112(3)$ & $1.206(9)$ & $0.3136(7)$ & $0.1154(5)$ & $1.1915(16)$ & $0.09(4)$ \\
\hline $\mathrm{C} 5$ & $-0.078(3)$ & $0.335(2)$ & $1.169(8)$ & $-0.0715(6)$ & $0.3372(7)$ & $1.1814(18)$ & $0.09(3)$ \\
\hline C6 & $0.231(5)$ & $0.341(3)$ & $0.715(15)$ & $0.2428(7)$ & $0.3383(6)$ & $0.7192(15)$ & $0.13(5)$ \\
\hline $\mathrm{C} 7$ & 0.0000 & 0.0000 & $1.410(12)$ & 0.0000 & 0.0000 & $1.4283(17)$ & $0.09(6)$ \\
\hline $\mathrm{O} 1$ & $0.041(3)$ & $0.087(3)$ & $1.531(8)$ & $0.0396(4)$ & $0.0847(3)$ & $1.5444(8)$ & $0.07(4)$ \\
\hline $\mathrm{O} 2 *$ & 0.0000 & 0.0000 & $1.140(10)$ & 0.0000 & 0.0000 & $1.1411(11)$ & 0.00 \\
\hline
\end{tabular}

* The origin of the structural model obtained from cRED data was placed to be the same as that of the model from SCXRD data, using the bridging $\mathrm{O} 2$ as a reference. 
Table 3. Comparison of the bond lengths and angles of BSA@ZIF-C refined against cRED data with those of ZIF$\mathrm{CO}_{3}-1$ refined against synchrotron SCXRD data.

\begin{tabular}{llll}
\hline Atoms 1,2 & $\mathrm{~d} 1,2(\AA)$ from cRED & $\mathrm{d} 1,2(\AA)$ from SCXRD & Difference d 1,2( $)$ \\
\hline $\mathrm{Zn} 1-\mathrm{N} 2$ & $1.97(3)$ & $1.972(5)$ & $0.00(3)$ \\
$\mathrm{Zn} 1-\mathrm{N} 3$ & $1.970(5)$ & $0.05(3)$ \\
$\mathrm{Zn} 1-\mathrm{O} 2$ & $2.02(3)$ & $1.982(3)$ & $0.03(2)$ \\
$\mathrm{Zn} 1-\mathrm{O} 1^{\mathrm{a}}$ & $1.95(2)$ & $1.988(4)$ & $0.05(4)$ \\
$\mathrm{C} 1-\mathrm{N} 3$ & $2.03(4)$ & $1.328(7)$ & $0.03(2)$ \\
$\mathrm{C} 1-\mathrm{N} 2^{\mathrm{b}}$ & $1.36(2)$ & $1.349(6)$ & $0.01(2)$ \\
$\mathrm{C} 4-\mathrm{N} 3$ & $1.34(2)$ & $1.376(9)$ & $0.02(3)$ \\
$\mathrm{C} 5-\mathrm{N} 2$ & $1.36(3)$ & $1.381(8)$ & $0.03(2)$ \\
$\mathrm{C} 4-\mathrm{C} 5^{\mathrm{b}}$ & $1.35(2)$ & $1.344(10)$ & $0.02(3)$ \\
$\mathrm{C} 1-\mathrm{C} 6$ & $1.36(3)$ & $1.487(8)$ & $0.02(5)$ \\
$\mathrm{O} 1-\mathrm{C} 7$ & $1.51(5)$ & $1.247(5)$ & $0.03(4)$ \\
$\mathrm{O} 2-\mathrm{C} 7$ & $1.28(4)$ & $1.338(9)$ & $0.08(6)$ \\
\hline Average & $1.26(6)$ & &
\end{tabular}

Average difference d 1,2( $\AA$ ) 0.04(3)

\begin{tabular}{llll} 
Atoms $1,2,3$ & Angle $1,2,3\left(^{\circ}\right)$ from cRED & Angle $1,2,3\left(^{\circ}\right)$ from SCXRD & Difference Angle $1,2,3\left(^{\circ}\right)$ \\
\hline $\mathrm{N} 2-\mathrm{Zn} 1-\mathrm{N} 3$ & $120.5(12)$ & $119.9(2)$ & $1(1)$ \\
$\mathrm{N} 2-\mathrm{Zn} 1-\mathrm{O} 2$ & $110.4(12)$ & $109.63(16)$ & $0(1)$ \\
$\mathrm{N} 2-\mathrm{Zn} 1-\mathrm{O} 1^{\mathrm{a}}$ & $107.8(15)$ & $108.00(18)$ & $0(2)$ \\
$\mathrm{N} 3-\mathrm{Zn} 1-\mathrm{O} 2$ & $108.8(10)$ & $111.60(15)$ & $3(1)$ \\
$\mathrm{N} 3-\mathrm{Zn} 1-\mathrm{O} 1^{\mathrm{a}}$ & $106.8(15)$ & $107.26(18)$ & $0(2)$ \\
$\mathrm{O} 2-\mathrm{Zn} 1-\mathrm{O} 1^{\mathrm{a}}$ & $100.6(15)$ & $98.11(18)$ & $3(2)$ \\
$\mathrm{N} 2^{\mathrm{b}}-\mathrm{C} 1-\mathrm{N} 3$ & $107(3)$ & $111.7(5)$ & $5(3)$ \\
$\mathrm{C} 1^{\mathrm{c}}-\mathrm{N} 2-\mathrm{C} 5$ & $107(3)$ & $106.0(5)$ & $1(3)$ \\
$\mathrm{C} 1-\mathrm{N} 3-\mathrm{C} 4$ & $112(3)$ & $105.4(5)$ & $7(3)$ \\
$\mathrm{N} 3-\mathrm{C} 4-\mathrm{C} 5^{\mathrm{b}}$ & $103(3)$ & $109.6(6)$ & $7(3)$ \\
$\mathrm{N} 2-\mathrm{C} 5-\mathrm{C} 4^{\mathrm{c}}$ & $112(3)$ & $107.3(6)$ & $5(3)$ \\
$\mathrm{N} 3-\mathrm{C} 1-\mathrm{C} 6$ & $126(3)$ & $123.9(5)$ & $2(3)$ \\
$\mathrm{N} 2^{\mathrm{b}}-\mathrm{C} 1-\mathrm{C} 6$ & $127(3)$ & $124.4(5)$ & $3(3)$ \\
$\mathrm{O} 1-\mathrm{C} 7-\mathrm{O} 1^{\mathrm{d}}$ & $128(6)$ & $128.6(7)$ & $1(6)$ \\
$\mathrm{O} 1-\mathrm{C} 7-\mathrm{O} 2$ & $116(3)$ & $115.7(4)$ & $0(3)$
\end{tabular}

Average difference angle $1,2,3\left(^{\circ}\right) 4(3)$

Symmetry transformations used to generate equivalent atoms: ${ }^{a} \mathrm{x}, \mathrm{y},-1+\mathrm{z} ;{ }^{\mathrm{b}} 0.5+\mathrm{x}, 0.5-\mathrm{y}, \mathrm{z} ;{ }^{\mathrm{c}}-0.5+\mathrm{x}, 0.5-\mathrm{y}, \mathrm{z} ;{ }^{\mathrm{d}}-\mathrm{x},-$ $\mathrm{y}, \mathrm{z}$.

\section{CONCLUSIONS}

Here, we demonstrate $a b$ initio structure determination of a biocomposite ZIF, BSA@ZIF-C by applying continuous rotation electron diffraction (cRED). We also show that the unit cell parameters can be accurately determined using cRED data. This is important for phase analysis and detection of possible structural changes in MOFs. The presence of BSA molecules in the crystal is indicated by a broad sphere 
in the 3D reciprocal space reconstructed from the cRED data, showing that the molecules are disordered. While it is not possible to locate the BSA molecules, the structure of ZIF-C could be solved and refined using the cRED data. We found that the structural model of ZIF-C obtained from the biocomposite BSA@ZIF-C is the same as that of ZIF-CO $\mathrm{CO}_{3}-1$ obtained by SCXRD. The encapsulation of the BSA in the ZIF does not make significant changes on the framework structure. We show that despite the high $R$ values originated from multiple scattering, accurate atomic structures can be obtained by cRED. The average deviation between the two models is 0.06 (4) $\AA$ for $\mathrm{Zn}(\mathrm{II})$ and $0.08(4) \AA$ for the light atoms. The combination of fast data collection and accurate structure determination by 3D ED opens new opportunities for studying the dynamical and cooperative structural changes in MOFs and other framework materials.

\section{Conflicts of interest}

There are no conflicts to declare.

\section{Acknowledgements}

This work was supported by the Swedish Research Council, with the project No. 2017-04321 (X.Z.) and 2016-04625 (Z.H). P. F. acknowledges TU Graz for the Lead Project (LP-03) and the European Union's Horizon 2020 Programme (FP/2014-2020)/ERC Grant Agreement No. 771834 - POPCRYSTAL.

\section{Notes and references}

(1) Yaghi, O. M.; Li, G.; Li, H. Nature 1995, 378 (6558), 703-706.

(2) Kitagawa, S.; Kitaura, R.; Noro, S. Angew. Chem. Int. Ed. 2004, 43 (18), 2334-2375.

(3) Furukawa, H.; Cordova, K. E.; O’Keeffe, M.; Yaghi, O. M. Science 2013, 341 (6149).

(4) Li, J.-R.; Sculley, J.; Zhou, H.-C. Chem. Rev. 2012, 112 (2), 869-932.

(5) Cui, Y.; Li, B.; He, H.; Zhou, W.; Chen, B.; Qian, G. Acc. Chem. Res. 2016, 49 (3), 483-493.

(6) Duan, J.; Jin, W.; Kitagawa, S. Coord. Chem. Rev. 2017, 332, 48-74.

(7) Bobbitt, N. S.; Mendonca, M. L.; Howarth, A. J.; Islamoglu, T.; Hupp, J. T.; Farha, O. K.; Snurr, R. Q. Chem. Soc. Rev. 2017, 46 (11), 3357-3385.

(8) Doonan, C.; Riccò, R.; Liang, K.; Bradshaw, D.; Falcaro, P. Acc. Chem. Res. 2017, 50 (6), 1423-1432.

(9) Horcajada, P.; Gref, R.; Baati, T.; Allan, P. K.; Maurin, G.; Couvreur, P.; Férey, G.; Morris, R. E.; Serre, C. Chem. Rev. 2012, 112 (2), 1232-1268.

(10) Park, K. S.; Ni, Z.; Côté, A. P.; Choi, J. Y.; Huang, R.; Uribe-Romo, F. J.; Chae, H. K.; O’Keeffe, M.; Yaghi, O. M. Proc. Natl. Acad. Sci. 2006, 103 (27), 10186-10191.

(11) Katsenis, A. D.; Puškarić, A.; Štrukil, V.; Mottillo, C.; Julien, P. A.; Užarević, K.; Pham, M.-H.; Do, T.-O.; Kimber, S. A. J.; Lazić, P.; et al. Nat. Commun. 2015, 6 (1), 1-8.

(12) Akimbekov, Z.; Katsenis, A. D.; Nagabhushana, G. P.; Ayoub, G.; Arhangelskis, M.; Morris, A. J.; Friščić, T.; Navrotsky, A. J. Am. Chem. Soc. 2017, 139 (23), 7952-7957.

(13) Chen, R.; Yao, J.; Gu, Q.; Smeets, S.; Baerlocher, C.; Gu, H.; Zhu, D.; Morris, W.; Yaghi, O. M.; Wang, H. Chem. Commun. 2013, 49 (82), 9500-9502.

(14) Kitagawa, S.; Uemura, K. Chem. Soc. Rev. 2005, 34 (2), 109-119.

(15) Schneemann, A.; Bon, V.; Schwedler, I.; Senkovska, I.; Kaskel, S.; Fischer, R. A. Chem. Soc. Rev. 2014, 43 (16), 6062-6096.

(16) Serre, C.; Mellot-Draznieks, C.; Surblé, S.; Audebrand, N.; Filinchuk, Y.; Férey, G. Science 2007, 315 (5820), $1828-1831$.

(17) Serre, C.; Millange, F.; Thouvenot, C.; Noguès, M.; Marsolier, G.; Louër, D.; Férey, G. J. Am. Chem. Soc. 2002, 124 (45), 13519-13526. 
(18) Lo, S.-H.; Feng, L.; Tan, K.; Huang, Z.; Yuan, S.; Wang, K.-Y.; Li, B.-H.; Liu, W.-L.; Day, G. S.; Tao, S.; et al. Nat. Chem. 2020, 12 (1), 90-97.

(19) Mason, J. A.; Oktawiec, J.; Taylor, M. K.; Hudson, M. R.; Rodriguez, J.; Bachman, J. E.; Gonzalez, M. I.; Cervellino, A.; Guagliardi, A.; Brown, C. M.; et al. Nature 2015, 527 (7578), 357-361.

(20) Yuan, S.; Zou, L.; Li, H.; Chen, Y.-P.; Qin, J.; Zhang, Q.; Lu, W.; Hall, M. B.; Zhou, H.-C. Angew. Chem. Int. Ed. 2016, 55 (36), 10776-10780.

(21) Su, J.; Yuan, S.; Wang, H.-Y.; Huang, L.; Ge, J.-Y.; Joseph, E.; Qin, J.; Cagin, T.; Zuo, J.-L.; Zhou, H.-C. Nat. Commun. 2017, 8 (1), 1-8.

(22) Moghadam, P. Z.; Ivy, J. F.; Arvapally, R. K.; Santos, A. M. dos; Pearson, J. C.; Zhang, L.; Tylianakis, E.; Ghosh, P.; Oswald, I. W. H.; Kaipa, U.; et al. Chem. Sci. 2017, 8 (5), 3989-4000.

(23) Dissegna, S.; Epp, K.; Heinz, W. R.; Kieslich, G.; Fischer, R. A. Adv. Mater. 2018, 30 (37), 1704501.

(24) Kirchon, A.; Feng, L.; Drake, H. F.; Joseph, E. A.; Zhou, H.-C. Chem. Soc. Rev. 2018, 47 (23), 8611-8638.

(25) Shearer, G. C.; Chavan, S.; Bordiga, S.; Svelle, S.; Olsbye, U.; Lillerud, K. P. Chem. Mater. 2016, 28 (11), 3749-3761.

(26) Vermoortele, F.; Bueken, B.; Le Bars, G.; Van de Voorde, B.; Vandichel, M.; Houthoofd, K.; Vimont, A.; Daturi, M.; Waroquier, M.; Van Speybroeck, V.; et al. J. Am. Chem. Soc. 2013, 135 (31), 11465-11468.

(27) Karagiaridi, O.; Bury, W.; Mondloch, J. E.; Hupp, J. T.; Farha, O. K. Angew. Chem. Int. Ed. 2014, 53 (18), $4530-4540$.

(28) Zheng, H.; Zhang, Y.; Liu, L.; Wan, W.; Guo, P.; Nyström, A. M.; Zou, X. J. Am. Chem. Soc. 2016, 138 (3), $962-968$.

(29) Groom, C. R.; Bruno, I. J.; Lightfoot, M. P.; Ward, S. C. Acta Crystallogr. Sect. B Struct. Sci. Cryst. Eng. Mater. 2016, $72(2), 171-179$.

(30) Belsky, A.; Hellenbrandt, M.; Karen, V. L.; Luksch, P. Acta Crystallogr. B 2002, 58 (Pt 3 Pt 1), 364-369.

(31) Gražulis, S.; Chateigner, D.; Downs, R. T.; Yokochi, A. F. T.; Quirós, M.; Lutterotti, L.; Manakova, E.; Butkus, J.; Moeck, P.; Le Bail, A. J. Appl. Crystallogr. 2009, 42 (4), 726-729.

(32) Dorset, D. L.; Hauptman, H. A. Ultramicroscopy 1976, 1 (3), 195-201.

(33) Dorset, D. L. Acta Crystallogr. A 1998, 54 (6), 750-757.

(34) Weirich, T. E.; Ramlau, R.; Simon, A.; Hovmöller, S.; Zou, X. Nature 1996, 382 (6587), 144-146.

(35) Kolb, U.; Gorelik, T.; Kübel, C.; Otten, M. T.; Hubert, D. Ultramicroscopy 2007, 107 (6), 507-513.

(36) Zhang, D.; Oleynikov, P.; Hovmöller, S.; Zou, X. Z Krist. 2010, 225, 94-102.

(37) Wan, W.; Sun, J.; Su, J.; Hovmöller, S.; Zou, X. J. Appl. Crystallogr. 2013, 46 (6), 1863-1873.

(38) Mugnaioli, E.; Gorelik, T.; Kolb, U. Ultramicroscopy 2009, 109 (6), 758-765.

(39) Boullay, P.; Palatinus, L.; Barrier, N. Inorg. Chem. 2013, 52 (10), 6127-6135.

(40) Shi, D.; Nannenga, B. L.; Iadanza, M. G.; Gonen, T. eLife 2013, 2, e01345.

(41) Cichocka, M. O.; Ångström, J.; Wang, B.; Zou, X.; Smeets, S. J. Appl. Crystallogr. 2018, 51 (6), 1652-1661.

(42) Gemmi, M.; La Placa, M. G. I.; Galanis, A. S.; Rauch, E. F.; Nicolopoulos, S. J. Appl. Crystallogr. 2015, 48 (3), 718-727.

(43) Nannenga, B. L.; Shi, D.; Leslie, A. G. W.; Gonen, T. Nat. Methods 2014, 11 (9), 927-930.

(44) Nederlof, I.; van Genderen, E.; Li, Y.-W.; Abrahams, J. P. Acta Crystallogr. D Biol. Crystallogr. 2013, 69 (7), 1223-1230.

(45) Yuan, S.; Qin, J.-S.; Xu, H.-Q.; Su, J.; Rossi, D.; Chen, Y.; Zhang, L.; Lollar, C.; Wang, Q.; Jiang, H.-L.; et al. ACS Cent. Sci. 2018, 4 (1), 105-111.

(46) Roy, S.; Huang, Z.; Bhunia, A.; Castner, A.; Gupta, A. K.; Zou, X.; Ott, S. J. Am. Chem. Soc. 2019, 141 (40), $15942-15950$.

(47) Carmona, F. J.; Maldonado, C. R.; Ikemura, S.; Romão, C. C.; Huang, Z.; Xu, H.; Zou, X.; Kitagawa, S.; Furukawa, S.; Barea, E. ACS Appl. Mater. Interfaces 2018, 10 (37), 31158-31167.

(48) Feyand, M.; Mugnaioli, E.; Vermoortele, F.; Bueken, B.; Dieterich, J. M.; Reimer, T.; Kolb, U.; de Vos, D.; Stock, N. Angew. Chem. Int. Ed. 2012, 51 (41), 10373-10376.

(49) Portolés-Gil, N.; Lanza, A.; Aliaga-Alcalde, N.; Ayllón, J. A.; Gemmi, M.; Mugnaioli, E.; López-Periago, A. M.; Domingo, C. ACS Sustain. Chem. Eng. 2018, 6 (9), 12309-12319.

(50) Denysenko, D.; Grzywa, M.; Tonigold, M.; Streppel, B.; Krkljus, I.; Hirscher, M.; Mugnaioli, E.; Kolb, U.; Hanss, J.; Volkmer, D. Chem. - Eur. J. 2011, 17 (6), 1837-1848.

(51) Wang, B.; Rhauderwiek, T.; Inge, A. K.; Xu, H.; Yang, T.; Huang, Z.; Stock, N.; Zou, X. Chem. - Eur. J. 2018, 24 (66), 17429-17433. 
(52) Yun, Y.; Wan, W.; Rabbani, F.; Su, J.; Xu, H.; Hovmöller, S.; Johnsson, M.; Zou, X. J. Appl. Crystallogr. 2014, 47 (6), 2048-2054.

(53) Wang, Y.; Yang, T.; Xu, H.; Zou, X.; Wan, W. J. Appl. Crystallogr. 2018, 51 (4), 1094-1101.

(54) Carraro, F.; Velasquez, M.; Astria, E.; Liang, W.; Twight, L.; Parise, C.; Ge, M.; Huang, Z.; Ricco, R.; Zou, X.; et al. 2019, doi: 10.26434/chemrxiv.10059935.

(55) Basnayake, S. A.; Su, J.; Zou, X.; Balkus, K. J. Inorg. Chem. 2015, 54 (4), 1816-1821.

(56) Stef Smeets; Bin Wang; Magdalena O. Cichocka; Jonas Ångström; Wei Wan. Zenodo, 2019.

(57) Wang, B.; Zou, X.; Smeets, S. IUCrJ 2019, 6 (5), 854-867.

(58) Zou, X. D.; Mo, Z. M.; Hovmoller, S.; Li, X. Z.; Kuo, K. H. Acta Crystallogr. A 2003, 59 (6), 526-539.

(59) Kabsch, W. Acta Crystallogr. D Biol. Crystallogr. 2010, 66 (2), 125-132.

(60) Kabsch, W. Acta Crystallogr. D Biol. Crystallogr. 2010, 66 (Pt 2), 133-144.

(61) Winter, G.; Waterman, D. G.; Parkhurst, J. M.; Brewster, A. S.; Gildea, R. J.; Gerstel, M.; Fuentes-Montero, L.; Vollmar, M.; Michels-Clark, T.; Young, I. D.; et al. Acta Crystallogr. Sect. Struct. Biol. 2018, 74 (2), 8597.

(62) Clabbers, M. T. B.; Gruene, T.; Parkhurst, J. M.; Abrahams, J. P.; Waterman, D. G. Acta Crystallogr. Sect. Struct. Biol. 2018, 74 (6), 506-518.

(63) Battye, T. G. G.; Kontogiannis, L.; Johnson, O.; Powell, H. R.; Leslie, A. G. W. Acta Crystallogr. D Biol. Crystallogr. 2011, 67 (4), 271-281.

(64) Sheldrick, G. M. Acta Crystallogr. A 2008, 64 (1), 112-122.

(65) Sheldrick, G. M. Acta Crystallogr. Sect. Found. Adv. 2015, 71 (1), 3-8.

(66) Karplus, P. A.; Diederichs, K. Science 2012, 336 (6084), 1030-1033. 
Structure determination by continuous rotation electron diffraction can be as feasible and accurate as single crystal X-ray diffraction without the need of large crystals.

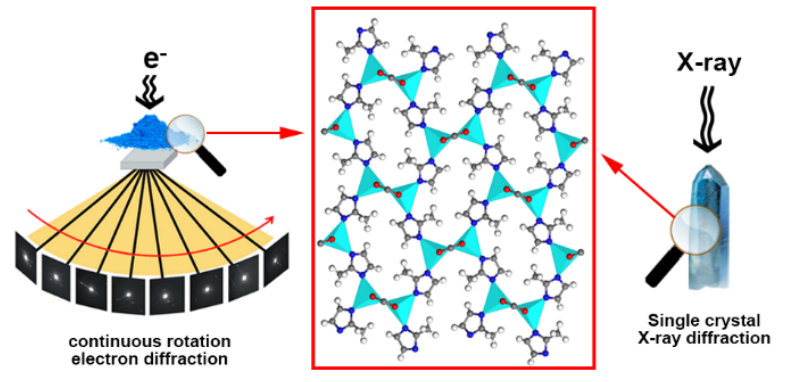

